\title{
Numerical Study of Nonlinear Dynamics of a Population System with Time Delay
}

\author{
Ivan N. Dushkov ${ }^{a}$, Ivan Jordanov ${ }^{b, c}$, Nikolay K. Vitanov ${ }^{c}$ \\ a Sofia University "St. Kl. Ohridski", Bulgaria, \\ ${ }^{b}$ University of National and World Economy, Sofia, Bulgaria, \\ ${ }^{c}$ Institute of Mechanics, Bulgarian Academy of Sciences
}

\begin{abstract}
Mathematical models of interacting populations are often constructed as systems of differential equations, which describe how populations change with time. Below we study one such model connected to the nonlinear dynamics of a system of populations in presence of time delay. The consequence of the presence of the time delay is that the nonlinear dynamics of the studied system become more rich, e.g., new orbits in the phase space of the system arise which are dependent on the time-delay parameters. In more detail we introduce a time delay and generalize the model system of differential equations for the interaction of three populations based on generalized Volterra equations in which the growth rates and competition coefficients of populations depend on the number of members of all populations [4, [5] and then numerically solve the system with and without time delay. We use a modification of the method of Adams for the numerical solution of the system of model equations with time delay. By appropriate selection of the parameters and initial conditions we show the impact of the delay time on the dynamics of the studied population system.
\end{abstract}

Keywords: Population dynamics; Generalized Volterra equations; Timedelay differential equations; Phase space; Adams method; Nonlinear dynamics; Chaos.

\section{Introduction}

Models based of differential and difference equations are widely used in many areas of natural and social sciences [7] [10], [12, [17] - [19], 21], 222, 24], 
[25], 29], [30], 42]- 49], [50]. Many of these models contain autonomous differential equations and because of this the theory of dynamical system gained a status of an essential tool for analysis of dynamics of many systems, e.g. in many areas of economic analysis particularly since computers has become commonly available [20],[27]. We note especially the application of theory of stochastic processes and dynamics with delay to different fields of science - fluid mechanics, economics, social sciences population dynamics and medicine [8], 11], [2], 26], [31] - [41]. The inclusion of time delay into the dynamic models leads to changes in their properties [14], 28]. Below we introduce time delay in the model of Dimitrova-Vitanov [3]- 6] and investigate the changes in the dynamics with increasing time delay. The model of Dimitrova and Vitanov without time delay describe competition and adaptation in a system of populations. Populations compete for resources and this competition often leads to significant changes in the number of individuals of populations. Populations react to changes by a greater or lesser adaptation. Those who adapt better, have better chances of survival. Clearly, it is important to model both processes simultaneously. In the model of Volterra the parameters describing the rate of growth and the rate of interaction among populations are constants. But the change in the number of members of populations leads to a change in the number of encounters between them. This can lead to a changes in the number of population members and the coefficients of the interaction. Thus the model of Dimitrova and Vitanov generalizes some classic models, such as the models of Volterra kind. From the point of view of mathematics the model equation in the Dimitrova-Vitanov model are ordinary differential equations. Below we shall introduce time delay in these equations and we shall assume that the time delay parameter is independent on the time.

\section{Mathematical formulation of the problem}

The generalized equation of Lotka - Voltera is:

$$
\frac{d N_{i}(t)}{d t}=r_{i} N_{i}(t)\left[1-\sum_{j=1}^{n} \alpha_{i j} N_{j}(t)\right], \quad i=1,2, \ldots, n,
$$

where $N_{i}(t)$ is the density of the members of the $i$-th competing population, $r_{i}$ is ratio of increase in the density of individuals of $i$-th population, and $\alpha_{i j}$ are the coefficients of the the interaction, showing how $j$-th population affects the $i$-th population. In this model, the coefficients representing the factors and interactions are constants. However, the change in the density of populations leads to a change in the number of encounters between 
population members. This in turn can lead to a change of both the ratios of increase and rates of interaction. This is a manifestation of the adaptation of the corresponding population systems to changes in environmental conditions. Considering this adaptation Dimitrova and Vitanov [3]- 6] have proposed the following relationships for the coefficients $r_{i}$ and $\alpha_{i j}$ of the members for each of the $\mathrm{n}$ competing populations:

$$
\begin{gathered}
r_{i}=r_{i}^{0}\left[1+\sum_{k=1}^{n} r_{i k} N_{k}+\sum_{k, l=1}^{n} r_{i k l} N_{k} N_{l}+\sum_{k, l, m=1}^{n} r_{i k l m} N_{k} N_{l} N_{m}+\ldots\right], \\
\alpha_{i j}=\alpha_{i j}^{0}\left[1+\sum_{k=1}^{n} \alpha_{i j k} N_{k}+\sum_{k, l=1}^{n} \alpha_{i j k l} N_{k} N_{l}+\sum_{k, l, m=1}^{n} \alpha_{i j k l m} N_{k} N_{l} N_{m}+\ldots\right] .
\end{gathered}
$$

Further Dimitrova and Vitanov have discussed the simplest, yet most natural form of the above relations, keeping only linear members. Thus, the relationships for the coefficients of the growth factors and the number of the reaction of members of the systems of agents become:

$$
\begin{aligned}
r_{i} & =r_{i}^{0}\left[1+\sum_{k=1}^{n} r_{i k} N_{k}\right], \\
\alpha_{i j} & =\alpha_{i j}^{0}\left[1+\sum_{k=1}^{n} \alpha_{i j k} N_{k}\right] .
\end{aligned}
$$

Taking into account (4) and (5) the system of ordinary differential equations for modeling the dynamics of system of interacting populations in the presence of adaptation becomes:

$$
\begin{array}{r}
\frac{d N_{i}}{d t}=r_{i}^{0} N_{i}\left\{1-\sum_{j=1}^{n}\left[\alpha_{i j}^{0}-r_{i j}\right] N_{j}-\sum_{j=1}^{n} \sum_{l=1}^{n} \alpha_{i j}^{0}\left[\alpha_{i j l}+\right.\right. \\
\left.\left.+r_{i l}\right] N_{j} N_{l}-\sum_{j=1}^{n} \sum_{k=1}^{n} \sum_{l=1}^{n} \alpha_{i j}^{0} r_{i k} \alpha_{i j l} N_{j} N_{k} N_{l}\right\} .
\end{array}
$$

The obtained model is a generalization of various models of dynamics of population. For an example if

$$
\begin{array}{r}
r_{i}^{0}=f_{i}^{0} \\
r_{i}^{0} \alpha_{i j}^{0}=-f_{i j}-b_{i j}^{0} \\
r_{i}^{0} \alpha_{i j}^{0}\left(\alpha_{i j k}+r_{i k}\right)=-b_{i j k} \\
r_{i}^{0} \alpha_{i j}^{0}+r_{i l} \alpha_{i j l} r_{i k}=0,
\end{array}
$$


then one obtains

$$
\frac{d N_{i}}{d t}=N_{i}\left[f_{i}^{0}+\sum_{j=1}^{n}\left(\alpha_{i j}^{0}+f_{i j}\right) N_{j}+\sum_{j, k=1}^{n} b_{i j k} N_{j} N_{l}\right] .
$$

Thus the model is reduced to the model discussed by Arneodo et al.[1], plus additional members, accounting for the adaptation effects.

Let us now introduce the constant time delay $\tau>0$ in the model. We obtain the following system of ordinary differential equations with time delay:

$$
\begin{array}{r}
\frac{d N_{i}(t)}{d t}=N_{i}(t-\tau)\left[f_{i}^{0}+\sum_{j=1}^{n}\left(\alpha_{i j}^{0}+f_{i j}\right) N_{j}(t-\tau)+\right. \\
\left.\sum_{j, k=1}^{n} b_{i j k} N_{j}(t-\tau) N_{l}(t-\tau)\right],
\end{array}
$$

or:

$$
\begin{array}{r}
\frac{d N_{i}(t)}{d t}=r_{i}^{0} N_{i}(t-\tau)\left\{1-\sum_{j=1}^{n}\left[\alpha_{i j}^{0}-r_{i j}\right] N_{j}(t-\tau)-\right. \\
\sum_{j=1}^{n} \sum_{l=1}^{n} \alpha_{i j}^{0}\left[\alpha_{i j l}++r_{i l}\right] N_{j}(t-\tau) N_{l}(t-\tau)- \\
\left.\sum_{j=1}^{n} \sum_{k=1}^{n} \sum_{l=1}^{n} \alpha_{i j}^{0} r_{i k} \alpha_{i j l} N_{j}(t-\tau) N_{k}(t-\tau) N_{l}(t-\tau)\right\} .
\end{array}
$$

Let us assume that the solution is sufficiently smooth and for the right side of the equations of the system we may use the decomposition in powers of $\tau$ :

$$
\begin{gathered}
\frac{d N_{i}(t)}{d t}=F_{i}(t-\tau) \\
F_{i}(t-\tau)=F_{i}(t)-\tau \frac{d F_{i}(t)}{d t}+\ldots+(-1)^{n} \frac{\tau^{n}}{n !} \frac{d^{n} F_{i}(t)}{d t^{n}}+\ldots,
\end{gathered}
$$

where:

$$
\begin{gathered}
\frac{d F_{i}(t)}{d t}=\sum_{j=1}^{n} \frac{\partial F_{i}}{\partial N_{j}} \frac{d N_{i}(t)}{d t} \\
\frac{d^{2} F_{i}(t)}{d t^{2}}=\sum_{j, k=1}^{n} \frac{\partial^{2} F_{i}}{\partial N_{j} \partial N_{k}} \frac{d N_{i}(t)}{d t} \frac{d N_{k}(t)}{d t}+\sum_{j=1}^{n} \frac{\partial F_{i}}{\partial N_{j}} \frac{d^{2} N_{i}(t)}{d t^{2}}
\end{gathered}
$$

Then we can discuss solutions to a system (10) as:

$$
N_{i}(t)=\sum_{\alpha=0}^{\infty}(-1)^{\alpha} \frac{\tau^{\alpha}}{\alpha !} N_{i, \alpha}(t)
$$


where $N_{i, \alpha}(t)$ are functions that must be determined by the sequence of equations for the different degrees of $\tau$ obtained from (10). For an example if $\alpha=0$ we obtain the solution without time delay. Obtained sequence of equations is quite complicated for analytical solution and because of this below we shall discuss numerical solution of the model system with time delay.

\section{Numerical solution}

We note that the presence of time delay reduces almost to 0 the possibility for analytical study of corresponding system of model equation. Because of this we have to solve numerically the corresponding equations. Below we discuss briefly our approach for numerical solution. First of all for the solution of the problem we need to set initial functions $\phi_{i}(t)$ in the range $[-\tau, 0]$. We will assume that these functions are the solution to the system, but without any time delay. In general the system we want to solve has the form:

$$
\frac{d N_{i}(t)}{d t}=F_{i}\left[N_{1}(t-\tau), \ldots, N_{n}(t-\tau)\right]
$$

Let us have the initial conditions $\phi_{i}(t)$ of the above kind defined in the interval $[-\tau, 0)$. We divide the interval of $k$ equal parts by the points $\{-\tau+l h\}$ where $l=0, \ldots, k$. Because of the requirement $-\tau+k h=0$ to step $h$ we receive $h=\tau / k$. Now we can find an approximation of the solution at the point $t=m h, m>0$. We integrate the above equations within the range of $t_{m-l}$ to $t_{m}$ :

$$
\int_{t_{m-l}}^{t_{m}} \dot{N}_{i}(t) d t=\int_{t_{m-l}}^{t_{m}} d N_{i}(t)=\int_{t_{m-l}}^{t_{m}} F_{i}\left[N_{1}(t-\tau), \ldots, N_{n}(t-\tau)\right] d t
$$

or

$$
N_{i}^{m}-N_{i}^{m-l}=\int_{t_{m-l}}^{t_{m}} F_{i}\left[N_{1}(t-\tau), \ldots, N_{n}(t-\tau)\right] d t
$$

Next we make the substitution $t=t_{m}+h x$. The result is:

$$
N_{i}^{m}-N_{i}^{m-l}=h \int_{-l}^{0} F_{i}\left[N_{1}\left(t_{m}+h x-\tau\right), \ldots, N_{n}\left(t_{m}+h x-\tau\right)\right] d x .
$$

Taking into account that

$$
F_{i}\left[N_{1}(t+h x-\tau), \ldots, N_{n}(t+h x-\tau)\right]=\frac{d N_{i}(t+h x)}{d t},
$$

we obtain:

$$
N_{i}^{m}-N_{i}^{m-l}=h \int_{-l}^{0} \dot{N}_{i}\left(t_{m}+h x\right) d x
$$


Now we apply Newton's formula for interpolating backwards (starting with point $t)$ :

$$
\begin{aligned}
\dot{N}_{i}\left(t_{m}+h x\right)= & \dot{N}_{i}^{m}+\frac{x}{1 !} \Delta \dot{N}_{i}^{m-1}+\frac{x(x+1)}{2 !} \Delta^{2} \dot{N}_{i}^{m-2}+\ldots \\
& +\frac{x(x+1) \ldots(x+k-1)}{k !} \Delta^{k} \dot{N}_{i}^{m-k}+R_{k}(x),
\end{aligned}
$$

where finite difference forward is

$$
\Delta^{l} \dot{N}_{i}=\sum_{l=0}^{k}(-1)^{k+l}\left(\begin{array}{l}
k \\
l
\end{array}\right) \dot{N}_{i}^{m-k+l},
$$

The result is

$$
\begin{array}{r}
\frac{N_{i}^{m+l}-N_{i}^{m-l}}{h}=\int_{-l}^{0}\left(\dot{N}_{i}^{m}+\frac{x}{1 !} \Delta \dot{N}_{i}^{m-1}+\right. \\
\left.\frac{x(x+1)}{2 !} \Delta^{2} \dot{N}_{i}^{m-2}+\frac{x(x+1) \ldots(x+k-1)}{k !} \Delta^{k} \dot{N}_{i}^{m-k}\right) d x+\int_{-l}^{0} R_{k}(x) d x .
\end{array}
$$

In order to obtain the value of the solution at the point $t_{m}$, we must use the values in the points $t_{m-j k}$, which is why when calculating the first approximation (for point 0), we can use the above method only when $l=1$. In order to obtain the $s$-th approximation we use the method for $l$ from 1 to $s$. Note that for $l=0$ the above method has a local error of approximation $O\left(h^{k+l}\right)$. We are interested in the change of the solution of the system in presence of a time delay as compared to the solution without presence of time delay. The system for the case without time delay is solved by the method of Runge-Kutta method of fourth order.

Let us now apply the above methodology. We are going to show results for a particular case of the discussed model system, namely for the system of three populations $(i=1,2,3)$

$$
\begin{gathered}
\frac{d N_{i}}{d t}=N_{i}\left[f_{i}^{0}+\sum_{j=1}^{3}\left(\alpha_{i j}^{0}+f_{i j}\right) N_{j}+\sum_{j, k=1}^{3} b_{i j k} N_{j} N_{l}\right], \\
b_{11}^{0}=-k_{1}, b_{12}^{0}-k_{1}, b_{13}^{0}=-k_{2}, b_{21}^{0}=k_{1}, b_{22}^{0}=k_{1}, \\
b_{23}^{0}=-k_{2}, b_{31}^{0}=-k_{3}, b_{32}^{0}=-k_{2}, b_{33}^{0}=-k_{3}, \\
b_{i j k}=b, f_{i j}=f, \\
f_{1}^{0}=2 k_{1}+k_{2}, f_{2}^{0}=-k_{1}, f_{3}^{0}=2 k_{2}+k_{3} .
\end{gathered}
$$


The fixed points of this model system are as follows [6]. The first fixed pointis

$$
N_{1}=\frac{-R_{1}\left(k_{1}+k 2\right)-3 k_{1}^{2}+2 k_{1} k_{2}+k_{2}^{2}}{2 k_{1}\left(k_{2}-k 1\right)}, N_{2}=\frac{R_{1}}{k_{2}-k_{1}}, N_{3}=0
$$

where $R_{1}$ is the real root of the equation:

$b Z^{2}+\left(2 k_{1}^{2}-6 b k_{1}-2 b k_{2}\right) Z+\left(2 k_{1}^{3}+2 k_{1}^{2} k_{2}+2 f k_{1} k_{2}+b k_{2}^{2}+6 f k_{1}^{2}+9 b k_{1}^{2}+6 b k_{1} k_{2}\right)$.

The second fixed point is:

$$
N_{1}=N_{2}=1, N_{3}=R_{2},
$$

where $R_{2}$ is the real root of the equation:

$$
b Z^{2}+\left(4 b+f-k_{2}\right) Z+2 f+4 b+k_{2} .
$$

If a time delay is presented then the system (19) is transformed to a system of the king (9). Some effects of presence of the time delay in the model equations may be illustrated as follows. First we consider the case without the presence of a time delay and choose the parameter values as follows: $k_{1}=0.5, k_{2}=0.1, k_{3}=1.05, f=10^{-6}, b=10^{-5}$. In this case the attractor in the phase space is a limit cycle. Let's include the time lag and gradually start to increase the value of the delay parameter. For small values of the time lag $\tau$ there is no qualitative change in the phase trajectory of the system. With increasing value of $\tau$ the behavior in phase space is transformed from limit cycle to a chaotic attrcator. For values of $\tau$ larger than 0.5 the solution is no more confined, which is related to reaching the limits of the stability of the numerical algorithm.

The presence of time delay may lead to a transition in the opposite direction: from chaotic to cyclic behaviour. In order to demonstrate this we choose the following values of parameters: $k_{1}=0.5, k_{2}=0.1, k_{3}=1.43$, $f=10^{-6}, b=10^{-5}$. In the case without delay the model system of equations describes chaotic behaviour in the phase space. For small values of the time lag the behaviour in the phase spaces remains chaotic, i.e., small value of the time lag is not sufficient to destabilize the chaotic attractor of the system. By increasing the value of the time lag the behavior in the phase space changes from chaotic to periodic one. When values of the time lag $\tau$ are approximately 0.01 a limit cycle appears that remains stable with increasing values of the time lag up to $\tau=0.5$. 


\section{Concluding Remarks}

In this paper, we discuss a nonlinear model of the dynamics of interacting populations with time delay. The models of kind (9) and (10) may have many applications as they take into an account not only the current state of the studied system but also account for the past states of the system. In addition these models account for the influence of environment on the growth rates as well as for the influence of the environment on the coefficients of the interaction among the studied populations. Because of this models like the model described above are very flexible and may be applied for description of phenomena in other areas such as biology, economy, technological evolution social sciences, etc. The presence of time delay complicates the model equation and what can be done is to perform numerical studies of the dynamics of corresponding system. We have shown that the method of Adams can be applied successfully for numerical solution of the model system of ordinary differential equations in the presence of a time delay. On the basis of the numerical studies one can draw several conclusions about the impact of the time lag on the behavior of the system. First of all and as it can be expected for small values of the time lag there is no substantial influence of the time lag on the kind of the attractor of the system in phase space. But the increasing values of the delay parameter may lead to such qualitative change in the behaviour. This change may be associated with both the transition from periodic to chaotic attractor and a transition from chaotic attractor to a limit cycle.

\section{Acknowledgements}

This work contains results, which are supported by the UNWE project for scientific researchers with grant agreement No. NID NI - 21/2016.

\section{References}

[1] Arneodo A., Coullet P., Tresser C. 1980 Occurrence of strange attractors in three-dimensional Volterra equations, Phys. Lett. A, 79, 259263.

[2] Demiray H. 1972 On the elasticity of soft biological tissues. J. Biomech. 5, 309-311.

[3] Dimitrova Z. I., Vitanov N. K. 2000 Influence of adaptation on the nonlinear dynamics of a system of competing populations, Physics Letters A 272 (5), 368-380. 
[4] Dimitrova Z. I., Vitanov N. K. 2001 Dynamical consequences of adaptation of the growth rates in a system of three competing populations, J. of Physics A: Mathematical and General 34(37), 7459-7473.

[5] Dimitrova Z. I., Vitanov N. K. 2001 Adaptation and its impact on the dynamics of a system of three competing populations, Physica A: Statistical Mechanics and its Applications 300 (1), 91-115.

[6] Dimitrova Z. I., Vitanov N. K. 2004 Chaotic pairwise competition, Theoretical Population Biology 66 (1), 1-12.

[7] Dimitrova ZI. 2012. On traveling waves in lattices: The case of Riccati lattices. Journal of Theoretical and Applied Mechanics 42, 3 - 22.

[8] Dimitrova ZI. 2015 Numerical investigation of nonlinear waves connected to blood flow in an elastic tube of variable radius. Journal of Theoretical and Applied Mechanics 45, 79-92.

[9] Elger DF, Blackketter DM, Budwig RS, Johansen KH. 1996 The influence of shape on the stresses in model abdominal aortic aneurysms. J Biomech Eng. 118, 326-332.

[10] Fowler AC. 1997 Mathematical Modeling in Applied Sciences Cambridge University Press, Cambridge, UK.

[11] Gopalakrishnan SS, Benoit P, Biesheuvel A. 2014 Dynamics of pulsatile flow through model abdominal aortic aneurysms. J. Fluid Mech. 758, 150-179.

[12] Haberman R. 1977 Mathematical Models. Mechanical Vibration, Population Dynamics and Traffic Flow. SIAM, Philadelphia, PA.

[13] Jeffrey A, Kawahara T. 1981 Asymptotic methods in nonlinear wave theory. Pitman, Boston.

[14] Kuang Y. 1993 Delay differential equations: with applications in population dynamics. Academic Press, Boston.

[15] Kudryashov NA. 2005 Simplest equation method to look for exact solutions of nonlinear differential equations. Chaos, Solitons \& Fractals 24, 1217 - 1231.

[16] Kudryashov NA, Loguinova NB 2008 Extended simplest equation method for nonlinear differential equations. Applied Mathematics and Computations 205, 396 - 402. 
[17] Martinov NK, Vitanov NK 1994 New class of running-wave solutions of the (2+1)-dimensional sine-Gordon equation. Journal of Physics A: Mathematical and General 27, 4611 - 4618.

[18] Martinov NK, Vitanov NK 1994 On self-consistent thermal equilibrium structures in twodimensional negative-temperature systems. Canadian Journal of Physics 72, 618 - 624 .

[19] Martinov N, Vitanov N 1992. Running wave solutions of the twodimensional sine-Gordon equation. Journal of Physics A: Mathematical and General 25, 3609 - 3613.

[20] Meiss JD . 2007 Differential Dynamical Systems. SIAM, Pliladelphia, PA.

[21] Murray JD. 2002 Mathematical Biology. Springer, New York.

[22] Panchev S, Spassova T., Vitanov NK. 2007. Analytical and numerical investigation of two families of Lorenz-like dynamical systems. Chaos, Solitons \& Fractals 33, 1658-1671

[23] Patel PJ, Greenfield JC, Fry DL. 1964 In vivo pressure length radius relationship in certain blood vessels in man and dog, in: E.O. Attinger (Ed.), Pulsatile Blood Flow, McGraw-Hill, New York, p. 277.

[24] Petrov V, Nikolova E, Timmer J. 2004. Dynamical analysis of cell function models. A review. Journal of Theoretical and Applied Mechanics 34, $55-78$.

[25] Petrov V, Nikolova E, Wolkenhauer O. 2007 Reduction of nonlinear dynamic systems with an application to signal transduction pathways. IET systems biology $\mathbf{1}, 2-9$.

[26] Prandtl L, Tietjens OG 1957 Applied Hydro- and Aeromechanics. Dover, New York.

[27] Starnberg S. 2013 Dynamical Systems. Dover, New York.

[28] Thomas E. 2009. Applied Delay Differential Equations. Springer, New York.

[29] Vitanov NK. 1996. On travelling waves and double-periodic structures in two-dimensional sine-Gordon systems. Journal of Physics A: Mathematical and General 29, 5195 - 5207 
[30] Vitanov NK, Dimitrova ZI, Kantz H. 2006 On the trap of extintion and its elimination. Physics Letters A 349, 350-355.

[31] Vitanov NK, Dimitrova ZI, Kantz H. 2010 Modified method of simplest equation and its application to nonlinear PDEs. Applied Mathematics and Computation 216, 2587 - 2595

[32] Vitanov NK. 2011 Modified method of simplest equation: Powerful tool for obtaining exact and approximate traveling-wave solutions of nonlinear PDEs. Communications in Nonlinear Science and Numerical Simulations 16, 1176 - 1185.

[33] Vitanov NK. 2011 On modified method of simplest equation for obtaining exact and approximate solutions of nonliear PDEs: the role of simplest equation. Communications in Nonlinear Science and Numerical Simulations 16, 4215 - 4231.

[34] Vitanov NK, Dimitrova ZI, Vitanov KN. 2015 Modified method of simplest equation for obtaining exact analytical solutions of nonlinear partial differential equations: further development of the methodology with applications. Applied Mathematics and Computation 269, 363 - 378

[35] Vitanov NK. 2010 Applications of simplest equations of Bernoulli and Riccati kind for obtaining exact traveling-wave solutions for a class of PDEs with polynomial nonlinearity. Communications in Nonlinear Sc ience and Numerical Simulation 15, 2050 - 2060.

[36] Vitanov NK, Dimitrova ZI. 2010 Application of the method of simplest equation for obtaining exact traveling-wave solutions for two classes of model PDEs from ecology and population dynamics. Communications in Nonlinear Science and Numerical Simulation 15, 2836 - 2845.

[37] Vitanov NK, Dimitrova ZI. 2014 Solitary wave solutions for nonlinear partial differential equations that contain monomials of odd and even grades with respect to partiacipating derivatives. Applied mathematics and Computation 247, 213 - 217.

[38] Vitanov NK, Dimitrova ZI, Vitanov KN. 2013 Traveling waves and statistical distributions connected to systems of interacting populations. Computers and Mathematics with Applications 66, 1666 - 1684.

[39] Vitanov NK, Dimitrova ZI, Kantz H. Application of the method of simplest equation for obtaining exact traveling-wave solutions for the extended Korteweg-deVries equation and generalized Camassa-Holm equation. Applied Mathematics and Computation 219, 7480 - 7492. 
[40] Vitanov NK, Dimitrova ZI, Vitanov KN. 2011 On the class of nonlinear PDEs that can b etreated by the modified method of simplest equation. Application to the generalized degasperis-Processi equation and $b$ equation. Communications in Nonlinear Science and Numerical Simulation 16, 1176 - 1185.

[41] Vitanov NK, Vitanov KN. 2014. Population dynamics in presence of state dependent fluctuations. Computers and Mathematics with Applications 68, 962 - 971.

[42] Vitanov NK, Dimitrova ZI, Ausloos M. Verhulst-Lotka-Volterra (VLV) model of ideological struggle. Physica A 389, 4970-4980.

[43] Vitanov NK. 2010. Optimum fields and bounds on heat transport for nonlinear convection in rapidly rotating fluid layer. The European Physical Journal B 73, 265 - 273.

[44] Vitanov NK. 2005. Upper bounds on convective heat transport in a layer of fluid of finite Prandtl number: Confirmation of Howards analytical asymptotic single-wave-number bound. Physics of Fluids 17, 105106.

[45] Vitanov NK. 1998. Bound on the heat transport in a horizontal fluid layer of infinite Prandtl number. Physics Letters A 248, 338 - 346.

[46] Vitanov NK, Busse FH. 1997. Bounds on the heat transport in a horizontal fluid layer with stress-free boundaries. Zeitschrift für Angewandte Mathematik und Physik ZAMP 48, 310 - 324.

[47] Vitanov NK, Ausloos M, Rotundo G. 2012 Discrete model of ideological struggle accounting for miigration. Advances in Complex Systems 15, 1250049 .

[48] Vitanov NK, Vitanov KN. 2016. Box model of migration channels. Mathematical Social Sciences 80, 108 - 114.

[49] Vitanov NK. 2016. Science dynamics and research production. Indicators, indexes, statistical laws and mathematical models. Springer International.

[50] Zhang WB. 2003 A Theory of Interregional Dynamics: Models of Capital, Kwowledge and Economic Structutes. Springer, Berlin. 